\title{
Successful treatment of a patient with brain metastases from endometrial cancer using Niraparib: a case report
}

\author{
Qing Wang, Fenghua Zhang, Hongyan Gao, Yun Xu \\ Department of Gynecology, Changzhou First People's Hospital, Changzhou, China \\ Correspondence to: Yun Xu. Department of Gynecology, Changzhou First People’s Hospital, 185 Juqian Street, Changzhou 213000 , China. \\ Email: xuyunbbb@163.com.
}

\begin{abstract}
Endometrial cancer (EC) is the second most common gynecologic malignancy in China, and the incidence and mortality rates have increased in recent years. Brain metastasis from EC is extremely rare, affecting only $0.3-1.16 \%$ of EC patients. The prognosis for patients with brain metastasis from EC is poor, with a median survival time of 3.5-6.5 months from the diagnosis of brain metastasis. Niraparib is a poly (adenosine diphosphate-ribose) polymerase (PARP) inhibitor that uses the concept of synthetic lethality in the presence of a mutation in the breast cancer susceptibility gene (BRCA). Niraparib is recommended as a maintenance treatment for ovarian cancer patients with platinum-sensitive relapse and has been shown to increase progression-free survival. Niraparib was found to enter the brain via the blood-brain barrier, which resulted in a higher concentration of the drug in the brain tissues and better tumor-suppressing effects. There was none report about the applications of PARP inhibitor for endometrial cancer with brain metastases. Here, we present the case of a 62-year-old woman whose Peripheral blood gene detection had shown BRCA1 mutation with brain metastases from high-grade serous carcinoma of the endometrium who was successfully treated with Niraparib and remained free of disease progression for 6 months.
\end{abstract}

Keywords: Case report; endometrial cancer (EC); brain metastasis; PARP inhibitors; Niraparib

Submitted Dec 02, 2020. Accepted for publication Jan 22, 2021.

doi: 10.21037/apm-21-113

View this article at: http://dx.doi.org/10.21037/apm-21-113

\section{Introduction}

Endometrial cancer (EC) is ranked as one of the three most common malignant tumors of the female genital tract. Statistics from the United States show that in 2019, there were 61,180 individuals living with EC and 12,160 associated deaths, placing it as the sixth leading cause of systemic tumor mortality (1). In China, EC is the second most common gynecologic malignancy after cervical cancer, with incidence and mortality rates of 63.4/100,000 and $21.8 / 100,000$, respectively (2) Furthermore, in recent years, the incidence and mortality of EC have shown an increasing trend. For the $70 \%$ of EC patients who are diagnosed with stage I disease, the recurrence rate is extremely low and the prognosis is usually good; however, for patients with advanced or specific pathological types of EC, the prognosis is poor. Previous studies have shown that while the 5 -year survival rate for endometrioid carcinoma is $83.2 \%$, this decreases to $28.6 \%$ for stage IV EC (3) and $52.6 \%$ for uterine serous carcinoma (USC) (4), respectively.

Metastasis of EC mainly manifests as local recurrence, which typically occurs in the pelvic cavity or para-aortic lymph nodes. Distant metastasis, however, is extremely rare and most frequently affects the liver, lung, and bones (5). Brain metastasis from EC has an incidence of only about $0.3-1.16 \%$ (6); however, the increase in the survival of EC patients in recent years has also seen a rise in the number of patients with brain metastases (7). Brain metastasis of EC has a poor prognosis, with a median survival time of only 3.5-6.5 months from the diagnosis of metastatic disease (6), and a 2 -year survival rate of $13.6 \%$. Previous studies have demonstrated that the median time from initial diagnosis of EC to brain metastasis is approximately 33 months. In 

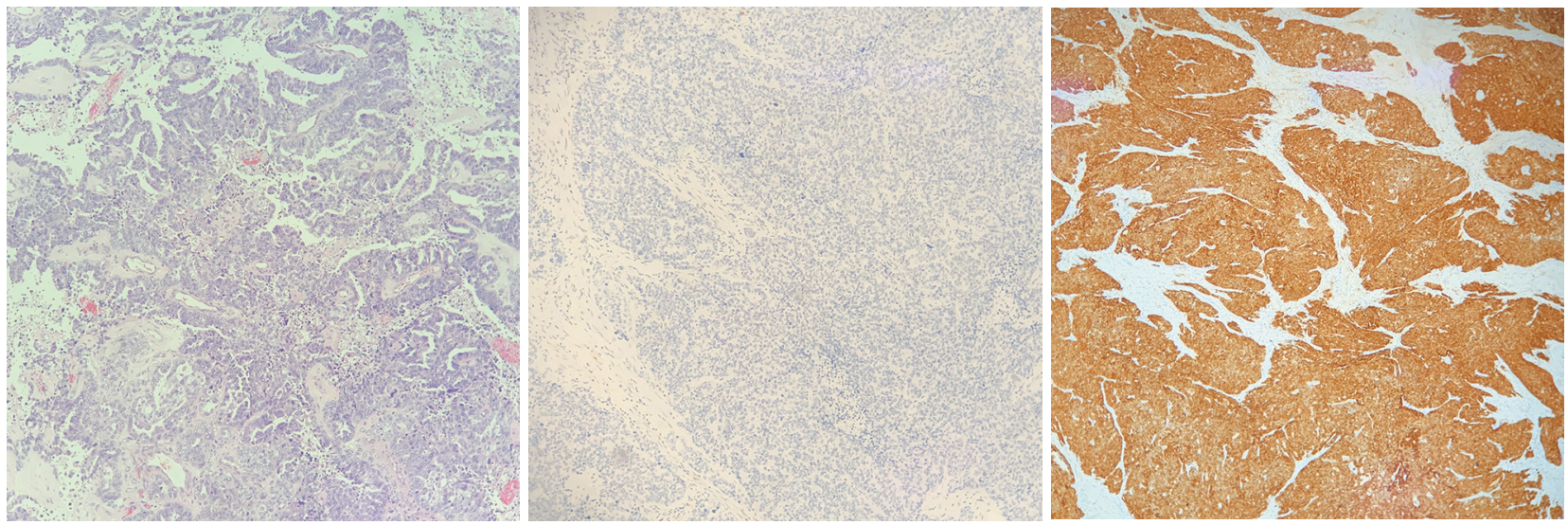

Figure 1 From left to right is postoperative pathological $(\mathrm{HE} \times 100)$, immunohistochemistry (p53 mutation) and immunohistochemistry (p16 positive).

Cohen et al.'s study (8), the mean survival time of patients with brain metastasis of EC was only about 1-2 months after the diagnosis of brain metastasis of EC. Therefore, the discovery of effective and appropriate treatments for EC patients with brain metastasis is vital. Although radiotherapy combined with surgery has been reported to effectively increase the survival rate of brain metastasis of EC, few studies to date have investigated treatments for patients with brain metastasis of EC, and the characteristics and best treatment regimens for such patients have yet to be described (9-18).

In a study of preclinical tumor models, Niraparib was found to enter the brain via the blood-brain barrier, which resulted in a higher concentration of the drug in the brain tissues and better tumor-suppressing effects (19).

We present the following article in accordance with the CARE reporting checklist (available at http://dx.doi. org/10.21037/apm-21-113).

\section{Case presentation}

A 62 -year-old woman with a body mass index of $21.23 \mathrm{~kg} / \mathrm{m}^{2}$ presented noticed abnormal vaginal bleeding in October 2017. The patient had previously been in good health, with no history of hypertension or diabetes, and no familial hereditary diseases. In October 2017, the patient underwent hysteroscopic endocervical curettage. Postoperative pathological examination suggested endometrial adenocarcinoma (Figure 1). Full abdominal computed tomography showed a space-occupying lesion in uterus $(3.1 \times 3.0 \times 3.0 \mathrm{~cm})$, as well as enlargement of the lymph nodes in the right iliac fossa. In November 2017, the patient underwent laparoscopic radical hysterectomy + bilateral adnexectomy + pelvic lymph node dissection + paraaortic lymph node dissection. Postoperative pathological examination showed high-grade USC invading the full thickness of uterus, and a cancerous embolus $(5 \times 3 \times 2 \mathrm{~cm})$ was found in the vessel. Immunohistochemistry revealed MS2(+), MSH6(+), MLH1(+), MSH2(+), ER(-), PR(-), p53(-), Ki67(70\%+), p16(+), and WT1(+). No obvious abnomalitis were observed in the internal cervical os or bilateral para-uterine tissues, nor did the bilateral oviducts or ovaries show any abnormalities. Metastasis was detected in the right pelvic lymph nodes (2/8), while the other lymph nodes showed reactive hyperplasia (left pelvic, 0/5; left common iliac, 0/4; right common iliac, $0 / 2$; and para-aortic, 0/2). Postoperative pathological staging was stage IIIC1 high-grade USC.

The patient subsequently underwent six cycles of chemotherapy with liposomal doxorubicin plus carboplatin (from November 25, 2017 to April 10, 2018). Postoperative follow-up showed a reduction in the patient's CA125 levels from $227.3 \mathrm{U} / \mathrm{mL}$ before the operation to $30.75 \mathrm{U} / \mathrm{mL}$ (normal level) in March 2018. Blood CA125 tests and imaging examinations were performed after the completion of chemotherapy, showing normal results.

In February 2019, the patient was admitted to the hospital with headache and myasthenia of the limbs. Cranial magnetic resonance imaging (MRI) examination showed multiple space-occupying lesions in the bilateral parietal-occipital lobes and right cerebellar hemisphere, which were considered as brain metastases, considering 

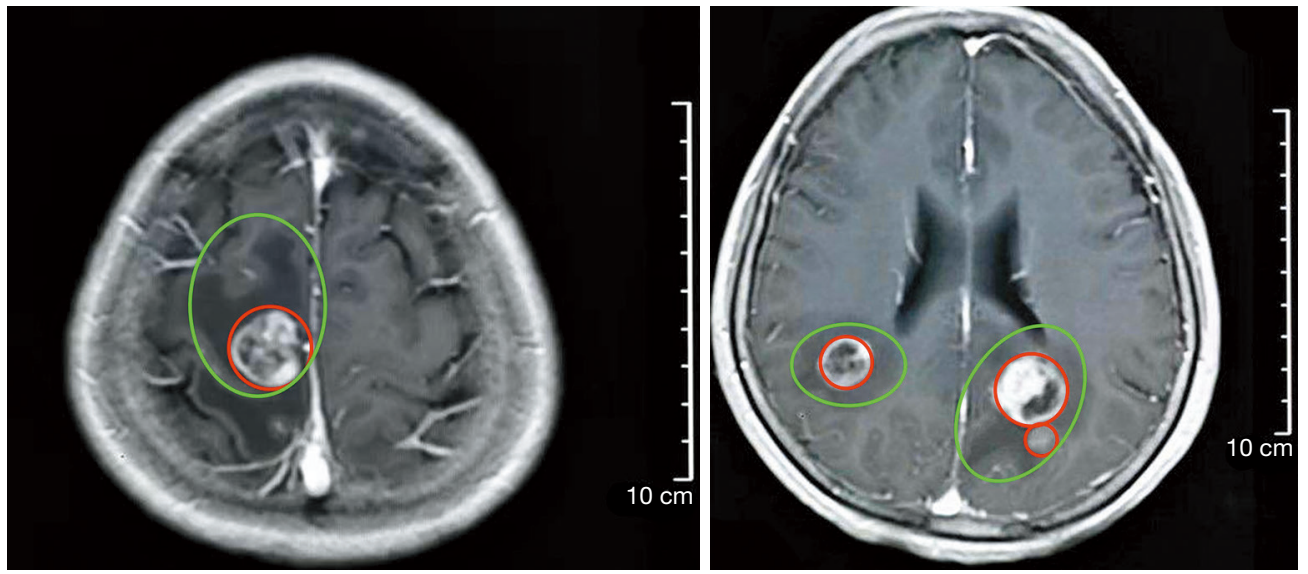

Figure 2 MRI of multiple intracranial metastases on March 7, 2019 (metastases circled in red; edema in green).
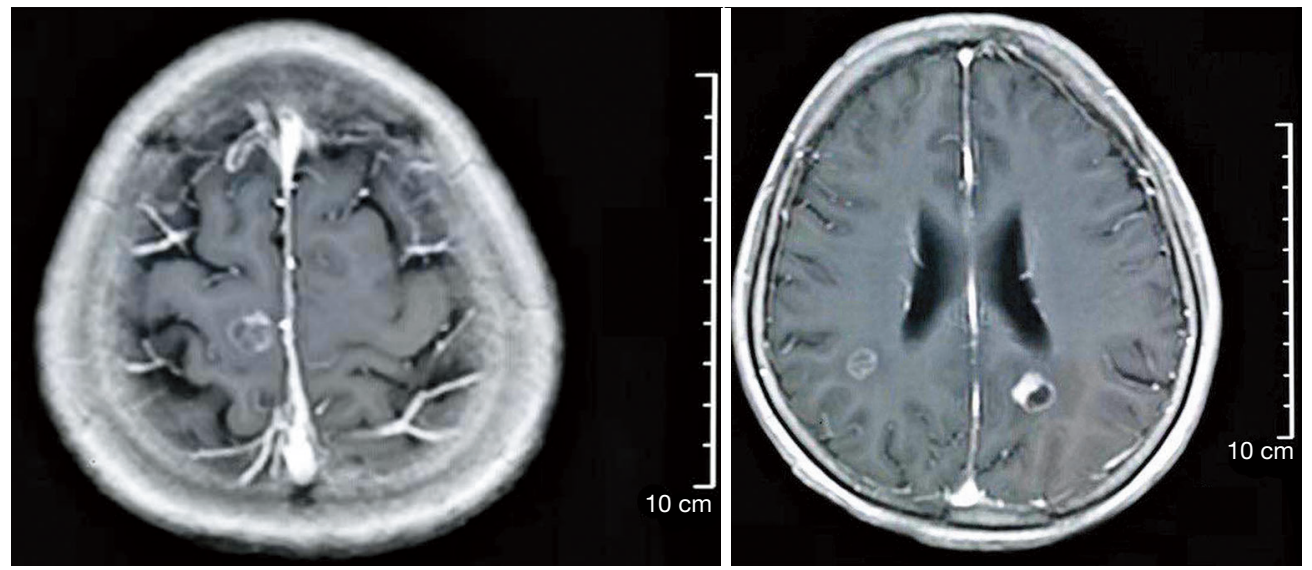

Figure 3 The intracranial metastatic lesions were reduced in size on May 9, 2019 compared to 2 years before, with the brain edema also reduced.

the patient's medical history (Figure 2). Whole-body positron emission tomography/computed tomography revealed post-EC surgery changes. The nodules in the right parietal lobe and bilateral occipital lobes showed abnormal 18F-fluorodeoxyglucose uptake accompanied by peri-nodular edema, which suggested brain metastases; no such abnormality was found in the abdominal cavity, retroperitoneum, or pelvic cavity. Also, the CA125 level in the patient's blood was increased to $38.4 \mathrm{U} / \mathrm{mL}$. Consequently, in March 2019, the patient was given wholebrain radiotherapy (WBRT) with a dose of $30 \mathrm{~Gy} / 10 \mathrm{fx}$, accompanied by $3 \mathrm{DRT}$ and oral administration of temozolomide $(150 \mathrm{mg} / \mathrm{d})$. On May 9 2019, the patient reported that the symptom of headache had improved substantially. Re-examination with cranial MRI showed that the lesions had shrunk compared to before treatment, and the peri-lesion edema was also evidently reduced (Figure 3). In July, however, the patient reported dizziness, distension in the head, astasia, and nausea, and cranial MRI examination was once again performed. Multiple intracranial brain metastases were detected. The largest lesion $(2.1 \times 1.5 \mathrm{~cm})$ was located at the left paracele, and another large lesion $(0.6 \times 0.4 \mathrm{~cm})$ was found at the left occipital lobe; the lesions were larger than those detected previously (Figure 4). Earlier microsatellite stability assessment (on March 15, 2019) had shown microsatellitestable disease. Peripheral blood gene detection in April 2019 had shown BRCA1 mutation. Therefore, on July 23, 2019, oral administration of Niraparib was initiated. As the bodyweight of the patient was $<77 \mathrm{~kg}$, an initial dose of 

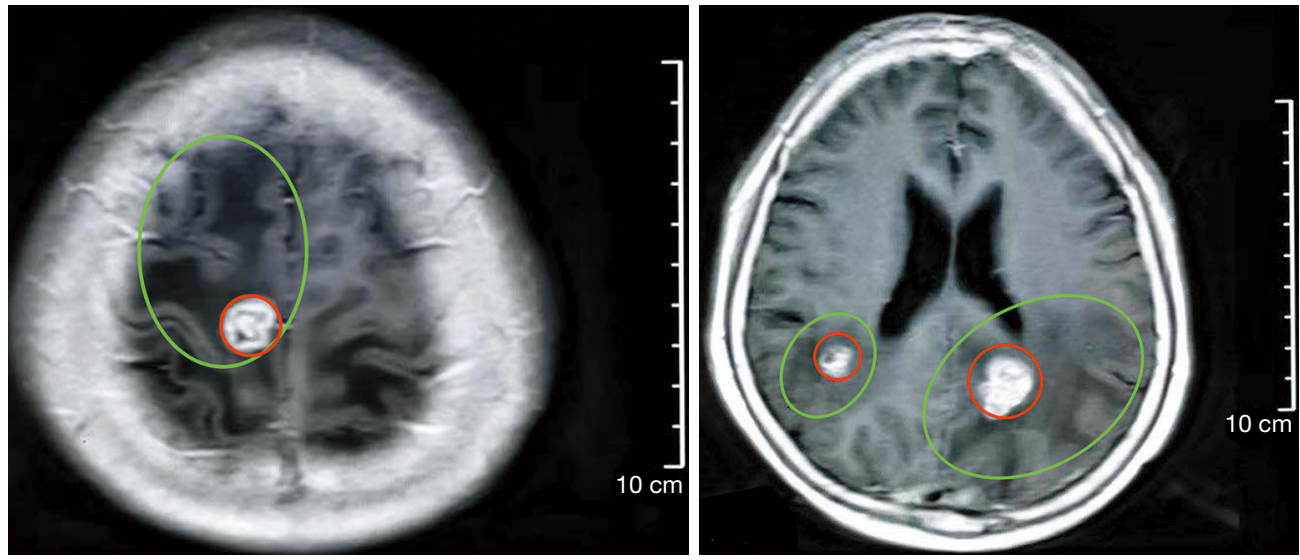

Figure 4 The intracranial metastatic lesions were increased in size and enlarged in the parenchymal tissues on August 7, 2019; peri-lesion edema was also evident, and the lesion in the left occipital lobe was enlarged (metastases circled in red; edema in green).
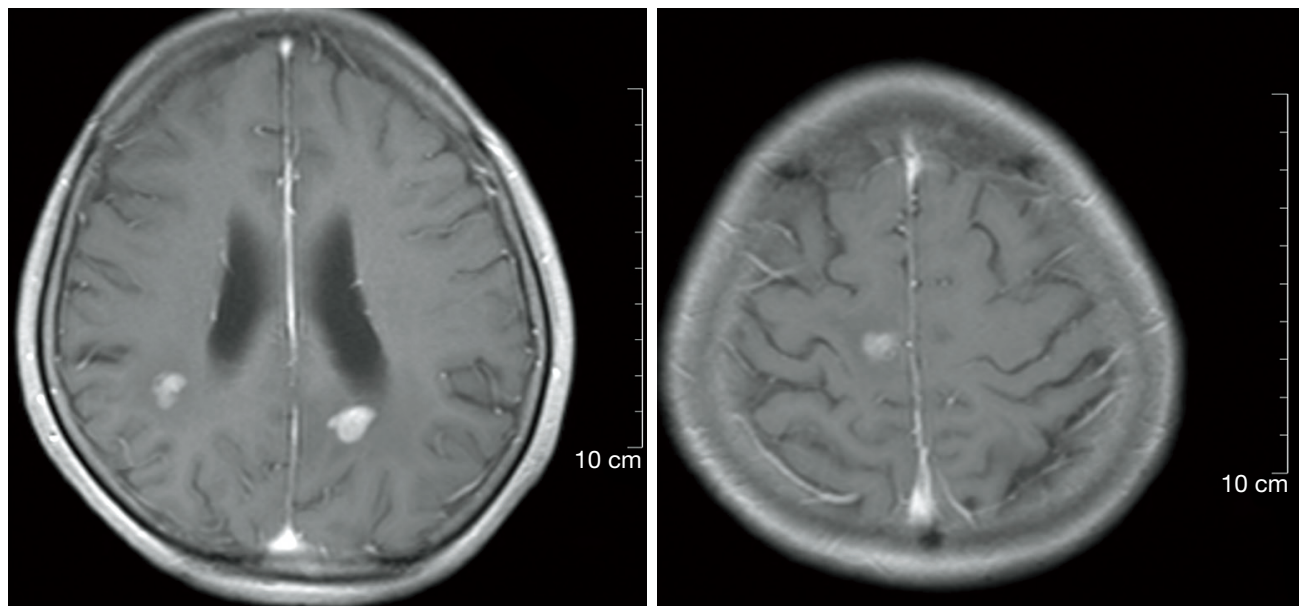

Figure 5 The multiple metastases in the bilateral parietal-occipital lobes and right cerebellar hemisphere were decreased in size, and the peri-lesion edema was reduced.

$200 \mathrm{mg}$ qd Niraparib was prescribed. Blood routine monitoring was performed every week during the first month of treatment, and once a month thereafter. During the treatment, only mild anemia (lowest hemoglobin level of $110 \mathrm{~g} / \mathrm{L}$ ) and platelet reduction (lowest value of $103 \times 10^{9} / \mathrm{L}$ ) were observed. The patient resumed normal walking and could perform her previous agricultural tasks by September 2019. She experienced no discomfort, such as headache or distension in the head. Reexamination on November 21 2019, showed the CA125 level to be $29.35 \mathrm{U} / \mathrm{mL}$. Cranial MRI examination on December 3 showed that the lesions had shrunk, the peri-lesion edema had ameliorated substantially, and the lesion of the left occipital lobe had shrunk substantially (Figure 5). Oral intake of Niraparib was continued until March 2020, at which point the patient again reported discomfort including headache (Figure 6). Reexamination by cranial MRI in the local hospital suggested new metastases in the right cerebellar hemisphere as well as a slight size increase in the metastases in the right parietal lobe, while no evident change of the metastases in the left parietal lobe and occipital lobe was observed (Figure 7).

Written informed consent was obtained from the patient for publication of this study and any accompanying images. All procedures performed in studies involving human participants were in accordance with the ethical standards of the institutional and/or national research committee(s) and with the Helsinki Declaration (as revised in 2013). 


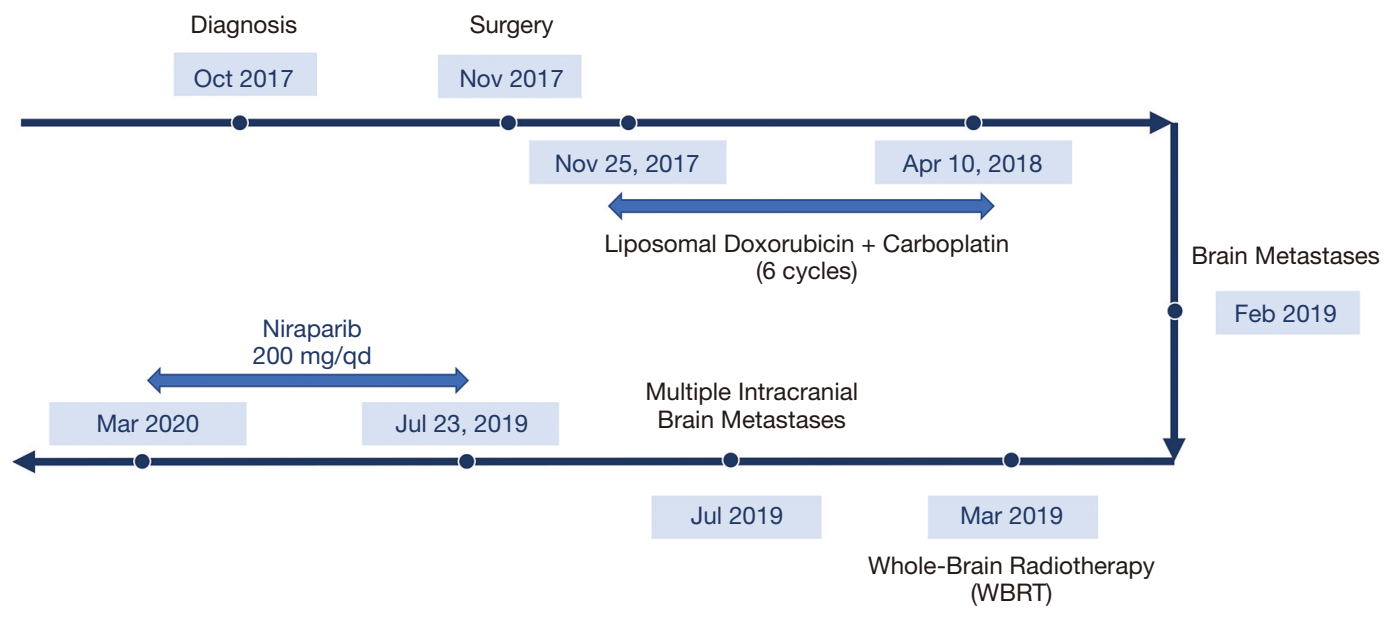

Figure 6 Case history.

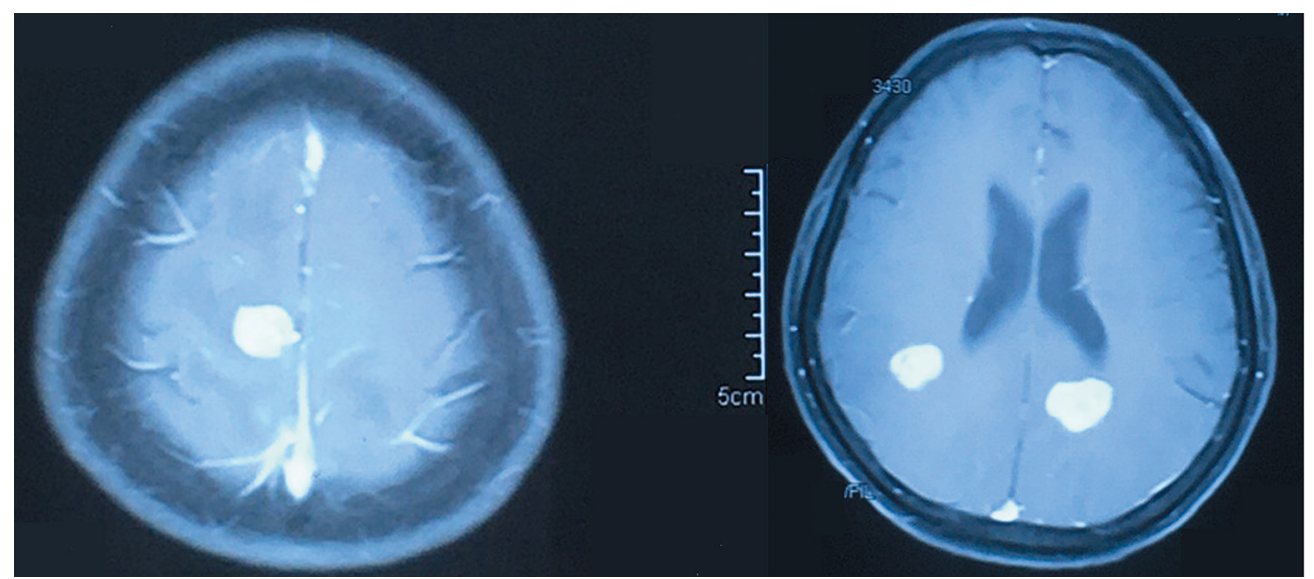

Figure 7 A new metastatic lesion appeared in the right cerebellar hemisphere in March 2020; the area of the metastases in the right parietal lobe was slightly increased, and no evident changes were found in the metastases in the left parietal or occipital lobes.

\section{Discussion}

\section{Brain metastasis of EC}

In cases of metastatic EC, the disease mainly spreads to the pelvic cavity and para-aortic lymph nodes via the lymphatic ducts. The second most common route of metastasis is hematogenous spread, mainly to the lung, liver, and bone (5). Brain metastasis of EC is extremely rare, but its occurrence is associated with a poor prognosis. To date, the risk factors for brain metastasis of EC have not been fully elucidated, nor have the optimal treatments for patients with this disease (20). Gien et al. (21) reported that about two-thirds of patients with brain metastases from EC had advanced disease (stage III/IV), and $80 \%$ of these patients had the high histological stage (G3). Moreover, Petru (22) reported the majority of the patients $(>90 \%)$ were found to have brain metastasis after the diagnosis of $\mathrm{EC}$, and the median time from initial diagnosis of EC to brain metastasis was 17 months. However, few patients $(<10 \%)$ were found to have brain metastasis before the diagnosis of EC. Brain metastasis of EC can involve a single lesion in the brain without metastases in other parts of the body, or multiple intracranial metastases with multiple metastases in other organs (23). Supratentorial lesions are the most common intracranial metastases in EC, accounting for $75 \%$ of all intracranial metastases in EC (20). The patient reported in the present case study was clinically diagnosed with stage IIIc1 EC, and histopathological examination showed G3 USC, which is a high-risk factor for brain 
metastasis. The time to brain metastasis after EC diagnosis and the initial EC treatment was 16 months and 10 months, respectively. The brain metastases manifested as multiple intracranial lesions and were not accompanied by multiple metastases in other parts of the body.

Patients with brain metastases of EC generally exhibit neurological symptoms, such as ataxia, unsteady gait, myasthenia of the limbs, hemiplegia, memory loss, paresthesia, diplopia, hearing disorders, and visual impairment (24). The patient in the present case study showed recurrence of the multiple intracranial lesions, which was accompanied by neurological symptoms including headache, numbness of the lower limbs, myasthenia of the limbs, and difficulty walking. These findings suggested that for EC patients with neurological symptoms, brain metastasis should be considered.

The treatment of brain metastasis has gradually expanded from WBRT alone to also include comprehensive treatment methods, such as surgical resection (craniotomy), stereotactic radiotherapy (SRT), molecular targeting treatment, and chemotherapy. Although brain metastases are the most common intracranial brain tumors, treatment methods vary according to the number, size, and location of the lesions, as well as the overall prognosis. As only a few cases have been reported, no standard treatment method exists for brain metastasis in patients with EC. Generally, such lesions are treated according to the general principles of neurological surgery. For instance, patients with a single metastatic brain lesion could undergo resection followed by WBRT, whereas for patients with multiple metastatic brain lesions, WBRT alone could be administered (5). SRT has clinical efficacy for the treatment of brain metastasis. Early detection of brain metastases is critical, as the lesions are generally small in the early stage with no evident peri-lesion edema and can be resected more easily. SRT also has fewer complications (25).

Gien et al. (21) reported that six of the eight patients in their study showed an improvement of neurological symptoms following treatment. However, the symptoms recurred in one patient 3 weeks later and in two other patients 1 month later (the data of the other three patients were not available). The symptoms of our patient also worsened at 2 months after WBRT, suggesting the recurrence of intracranial metastatic lesions.

\section{Application of poly (adenosine diphosphate-ribose) polymerase (PARP) inbibitors}

Mechanisms involved in the effects of PARP inhibitors PARP is a non-histone chromosomal protein in eukaryotes, which plays a critical role in DNA damage repair. PARP enzymes can recognize and repair single-strand DNA damage. If single-strand DNA damage is not repaired, it progresses to double-strand DNA damage. In normal cells, double-strand DNA damage can be repaired via homogenous recombination (HR) pathways, and cells can still survive after the DNA damage has been repaired. However, simultaneous dysfunction of PARP-dependent single-strand DNA damage repair and HR repair results in cell death; this has been described as the "synthetic lethality" effect (26). In tumor cells with HR defects, such as cells with breast cancer susceptibility gene (BRCA) 1/2 gene mutations, the HR pathway-mediated repair of doublestrand DNA damage is not activated. PARP inhibitors can then block the repair of single-strand DNA damage, thus inducing synthetic lethality and subsequent tumor cell apoptosis. Meanwhile, in normal cells, homologous recombination repair (HRR) functions normally; thus, DNA damage can be repaired, even in the presence of PARP inhibitors, preventing cell apoptosis. Therefore, PARP inhibitors can induce the synthetic lethality effect to selectively bring about the death of HRR-deficient cells.

Various genes participate in the HRR process. For instance, mutations of ATM, ATRX, ARID1A, and CHECK2, as well as hypermethylation of the RAD51C gene, amplification of the EMSY gene, and deletion or mutation of the PTEN gene possibly induce HRR dysfunction in cells (27). Therefore, PARP inhibitors could exert a tumor-killing effect in tumor cells with such genetic change. Heeke et al. (28) used next-generation sequencing (NGS) to analyze HRR-related genes in 52,426 solid tumors; in the $1,475 \mathrm{EC}$ patients involved, the mutation rate of HRR-related genes was $34.4 \%$, meaning EC ranked first among these solid tumors. Mutations of ARID1A were found to be the most common mutations in EC, accounting for $27.45 \%$ of all the HRR-related gene mutations. Furthermore, the total mutation rate of BRCA1 and BRCA2 was only $4.34 \%$ in EC. Therefore, EC is the tumor with the highest prevalence of HRR gene mutations, and thus, PARP inhibitors could play an important role in treating EC.

Evidence from clinical practice shows that $70-80 \%$ of EC patients have deletion of the tumor suppressor gene PTEN (29). Previous studies have demonstrated that the PTEN protein can influence the HR-mediated repair of double-strand DNA breaks and cell cycle checkpoints, and thus plays an important role in maintaining genetic stability. Based on these findings, the PARP inhibitor olaparib 
was used to treat two EC patients with PTEN defects and achieved good efficacy (30). Two in vitro studies also demonstrated that PARP inhibitors have killing effects on EC cell lines with PTEN dysfunction $(31,32)$. Currently, PARP inhibitors are being investigated in several clinical trials of targeted therapies for EC. PARP inhibitors are potentially important drugs to increase the clinical efficacy of chemotherapy and radiotherapy in patients with EC.

\section{Types of PARP inhibitors}

The PARP family includes 17 members, of which PARP1 is the most widely investigated. PARP1 accounts for over $90 \%$ of the PARP family functions in cells. PARP1 inhibitors were initially used as sensitizers in chemotherapy and radiotherapy treatment of cancer patients, as they can inhibit DNA repair in tumor cells damaged by radiotherapy or chemotherapy to exert synergetic anti-cancer effects. However, with the introduction of the concept of synthetic lethality, it is possible to treat cancers with BRCA gene mutations with monotherapy with PARP1 inhibitors (33). In December 2014, olaparib, which was developed by AstraZeneca, was approved by the United States Food and Drug Administration (US FDA). As the first small-molecular PARP1/2 inhibitor to be approved, olaparib achieved satisfactory effects in the treatment of breast cancers and ovarian cancers with BRCA1/2 gene defects $(34,35)$. In December 2016, rucaparib, another PARP1/2 inhibitor, was also approved for the treatment of advanced ovarian cancer patients with BRCA mutations who have received $\geq 2$ lines of chemotherapies (36). Shortly after, Niraparib was approved by the US FDA in March 2017 for the treatment of recurrent epithelial ovarian cancer, fallopian tube carcinoma, and primary peritoneal cancer, as well as for maintenance therapy in adult patients with complete or partial response and without BRCA genotype measurement results. Talazoparib, the most powerful PARP1 inhibitor currently, was approved in October 2018 for the treatment of locally advanced or metastatic HER2-negative breast cancers with BRCA mutations (37).

\section{Niraparib}

Niraparib, which was approved in 2017, is a highly selective powerful PARP-1 and -2 inhibitor used as monotherapy for patients with tumors with HR repair defects. It can also be combined with cytotoxic agents and radiotherapy as a sensitizer, or with immunological anti-tumor biological agents.

Niraparib has been or is being evaluated in 17 phase I-
III clinical studies performed by TESARO. As of August 2019, 1,653 patients had been treated with at least one dose of Niraparib by TESARO; these patients comprised the safety analysis set. The highest dose in the phase I trial was $400 \mathrm{mg}$ QD, the dose-limiting toxicity (DLT) of which was thrombocytopenia. The recommended dose of Niraparib in the phase II trial was $300 \mathrm{mg}$ QD, which was subsequently approved as a maintenance therapy for adult patients with recurrent epithelial ovarian cancer, fallopian tube carcinoma, and primary peritoneal carcinoma who achieved complete remission or partial remission after platinumbased chemotherapy.

Data about the effectiveness and safety of Niraparib as a maintenance therapy for patients with recurrent epithelial ovarian cancer with sensitivity to platinum were mainly obtained in the stage III NOVA study (37). The study included 546 patients treated with Niraparib or a placebo. The findings showed that daily oral intake of Niraparib prolonged the effects of platinum-based chemotherapy in a large number of patients, improved the progression-free survival, and reduced the risk of recurrence or death.

In a study of preclinical tumor models, Niraparib was found to enter the brain via the blood-brain barrier, which resulted in a higher concentration of the drug in the brain tissues and better tumor-suppressing effects. However, the same effects were not observed in the olaparib group (19). Based on these findings and the examination results of BRCA1 mutation, we selected Niraparib for the treatment of our patient, who experienced disease recurrence after developing brain metastasis of EC. The treatment showed clinical efficacy, with the patient achieving progression-free survival lasting 6 months.

With the wide application of PARP inhibitors in patients with ovarian and breast cancer patients, the safety and clinical efficacy of PARP inhibitors have been further verified. Although various PARP1/2 inhibitors have been approved to date, these drugs generally have low subtype selectivity; therefore, they could also inhibit the activity of PARP2 as well as that of PARP1, thus inducing side effects such as chronic anemia.

\section{Prospective PARP inhibitors for the treatment of EC}

With the wide application of PARP in treating ovarian and breast cancers, the safety and clinical efficacies of PARP inhibitors have also been further demonstrated, while monotherapy or combined therapy of PARP inhibitors for $\mathrm{EC}$ is still being investigated in clinical trials.

Gockley et al. (38) reported the first case of recurrent 
EC treated with olaparib in 2018. The patient had highly differentiated, stage IA endometrioid adenocarcinoma and underwent laparoscopic total hysterectomy (having previously received bilateral adnexectomy). Postoperative pathological examination revealed that the tumor was $3.1 \mathrm{~cm}$ in size. The patient had no other high-risk factors and had not been treated with adjuvant therapy. Chemotherapy, radiotherapy, and re-operation were performed on the patient after multiple recurrences, and an allergy to carboplatin was found during chemotherapy. Genetic examination of the patient showed germline and somatic BRCA2 mutations; thus, the patient was prescribed olaparib monotherapy (300 mg, twice a day, oral intake). Magnetic resonance imaging after 10 months of olaparib treatment showed that the size of the local tumor had reduced from $5.6 \times 3.9 \mathrm{~cm}$ to $2.2 \times 1.5 \mathrm{~cm}$, and the tumor remained stable for 15 months. An open-label phase II clinical trial (NCT03016338) of Niraparib for recurrent EC was initiated in November 2017 and is currently ongoing. The trial, which was designed to include 44 patients, will be completed in September 2022. Another multicenter, double-blind, randomized, phase II clinical trial (NCT03745950) of olaparib for the treatment of platinumsensitive advanced EC was initiated in February 2019. The trial includes 147 patients, divided into the study group and placebo group, and will be completed in December 2024. The findings of these studies are highly anticipated.

Multiple clinical studies are currently being performed to investigate the treatment of EC using the combination of PARP inhibitors with PI3K/AKT/mTOR pathway inhibitors, anti-angiogenesis agents, and immune checkpoint agents. More studies are needed on the clinical efficacy, safety, and biomarkers of EC treatment, as are studies to determine the patients who will best benefit, the best drug combinations, and the health-economic effectiveness.

\section{Conclusions}

Despite its low incidence, brain metastasis from EC has a poor prognosis and substantially reduces the quality of life of patients. Niraparib was selected for the treatment of the patient in the present case study due to the following reasons: (I) the patient had a gBRCA1 mutation; and (II) previous in vitro studies have demonstrated that Niraparib can pass through the blood-brain barrier. The patient is still taking Niraparib treatment and had achieved progression-free survival lasting 6 months ended in March
2020; moreover, her quality of life has been substantially increased. The patient could not walk before the treatment but has since regained the ability to stand, and is even performing daily activities and agricultural tasks. No evident side-effects have been observed. Therefore, we can proudly announce that this is a successful case of the treatment of brain metastasis in a patient with EC. However, due to the re-progression of the intracranial metastatic lesions, the patient underwent combination therapy of Niraparib plus an anti-angiogenesis agent, which helped to regain clinical remission.

\section{Patient perspective}

The patient felt desperate when she was informed multiple intracranial brain metastases. But things took a turn when she started taking Niraparib. She was grateful that we had tried.

\section{Acknowledgments}

Funding: None.

\section{Footnote}

Reporting Checklist: The authors have completed the CARE reporting checklist. Available at http://dx.doi.org/10.21037/ apm-21-113

Conflicts of Interest: All authors have completed the ICMJE uniform disclosure form (available at http://dx.doi. org/10.21037/apm-21-113). The authors have no conflicts of interest to declare.

Ethical Statement: The authors are accountable for all aspects of the work in ensuring that questions related to the accuracy or integrity of any part of the work are appropriately investigated and resolved. Written informed consent was obtained from the patient for publication of this study and any accompanying images. All procedures performed in studies involving human participants were in accordance with the ethical standards of the institutional and/or national research committee(s) and with the Helsinki Declaration (as revised in 2013).

Open Access Statement: This is an Open Access article distributed in accordance with the Creative Commons Attribution-NonCommercial-NoDerivs 4.0 International License (CC BY-NC-ND 4.0), which permits the non- 
commercial replication and distribution of the article with the strict proviso that no changes or edits are made and the original work is properly cited (including links to both the formal publication through the relevant DOI and the license). See: https://creativecommons.org/licenses/by-nc-nd/4.0/.

\section{References}

1. Siegel RL, Miller KD, Jemal A. Cancer statistics, 2019. CA Cancer J Clin 2019;69:7-34.

2. Chen $\mathrm{W}$, Zheng R, Baade PD, et al. Cancer statistics in China, 2015. CA Cancer J Clin 2016;66:115-32.

3. Tai YJ, Hsu HC, Chiang YC, et al. Impact of Adjuvant Modalities on Survival in Patients with Advanced Stage Endometrial Carcinoma: A Retrospective Analysis from a Tertiary Medical Center. Int J Environ Res Public Health 2019;16:2561.

4. Ouyang C, Frimer M, Hou LY, et al. Malignant Endometrial Polyps in Uterine Serous Carcinoma: The Prognostic Value of Polyp Size and Lymphovascular Invasion. Int J Gynecol Cancer 2018;28:524-8.

5. Piura E, Piura B. Brain metastases from endometrial carcinoma. ISRN Oncol 2012;2012:581749.

6. Moroney MR, Wheeler LJ, Corr BR. Clinical presentation of brain metastases from endometrial carcinoma: A case series. Gynecol Oncol Rep 2019;28:79-83.

7. Kim YZ, Kwon JH, Lim S. A clinical analysis of brain metastasis in gynecologic cancer: a retrospective multiinstitute analysis. J Korean Med Sci 2015;30:66-73.

8. Cohen ZR, Suki D, Weinberg JS, et al. Brain metastases in patients with ovarian carcinoma: prognostic factors and outcome. J Neurooncol 2004;66:313-25.

9. Cormio G, Lissoni A, Losa G, et al. Brain metastases from endometrial carcinoma. Gynecol Oncol 1996;61:40-3.

10. Wroński M, Zakowski M, Arbit E, et al. Endometrial cancer metastasis to brain: report of two cases and a review of the literature. Surg Neurol 1993;39:355-9.

11. Ruelle A, Zuccarello M, Andrioli G. Brain metastasis from endometrial carcinoma. Report of two cases. Neurosurg Rev 1994;17:83-7.

12. Sewak S, Muggia FM, Zagzag D. Endometrial carcinoma with cerebellar metastasis: a case report and review of the literature. J Neurooncol 2002;58:137-40.

13. Martínez-Mañas RM, Brell M, Rumia J, et al. Brain metastases in endometrial carcinoma. Gynecol Oncol 1998;70:282-4.

14. Kottke-Marchant K, Estes ML, Nunez C. Early brain metastases in endometrial carcinoma. Gynecol Oncol
1991;41:67-73.

15. Mahmoud-Ahmed AS, Suh JH, Barnett GH, et al. The effect of radiation therapy on brain metastases from endometrial carcinoma: a retrospective study. Gynecol Oncol 2001;83:305-9.

16. Sawada M, Inagaki M, Ozaki M, et al. Long-term survival after brain metastases from endometrial carcinoma. Jpn J Clin Oncol 1990;20:312-5.

17. Petru E, Lax S, Kurschel S, et al. Long-term survival in a patient with brain metastases preceding the diagnosis of endometrial cancer: report of two cases and review of the literature. J Neurosurg2001;94:846-8.

18. Shiohara S, Ohara M, Itoh K, et al. Successful treatment with sterotactic radiosurgery for brain metastases of endometrial carcinoma: a case report and review of the literature. Int J Gynecol Cancer 2003;13:71-6.

19. Sun K, Mikule K, Wang Z, et al. A comparative pharmacokinetic study of PARP inhibitors demonstrates favorable properties for niraparib efficacy in preclinical tumor models. Oncotarget 2018;9:37080-96.

20. Uccella S, Morris JM, Multinu F, et al. Primary brain metastases of endometrial cancer: A report of 18 cases and review of the literature. Gynecol Oncol 2016;142:70-5.

21. Gien LT, Kwon JS, D'Souza DP, et al. Brain metastases from endometrial carcinoma: a retrospective study. Gynecol Oncol 2004;93:524-8.

22. Petru E, Lax S, Kurschel S, et al. Long-term survival in a patient with brain metastases preceding the diagnosis of endometrial cancer. Report of two cases and review of the literature. J Neurosurg 2001;94:846-8.

23. Mahmoud-Ahmed AS, Suh JH, Barnett GH, et al. The effect of radiation therapy on brain metastases from endometrial carcinoma: a retrospective study. Gynecol Oncol 2001;83:305-9.

24. Monaco E, 3rd, Kondziolka D, Mongia S, et al. Management of brain metastases from ovarian and endometrial carcinoma with stereotactic radiosurgery. Cancer 2008;113:2610-4.

25. Kasper E, Ippen F, Wong E, et al. Stereotactic radiosurgery for brain metastasis from gynecological malignancies. Oncol Lett 2017;13:1525-8.

26. Thompson JM, Nguyen QH, Singh M, et al. Approaches to identifying synthetic lethal interactions in cancer. Yale J Biol Med 2015;88:145-55.

27. Wurster S, Hennes F, Parplys AC, et al. PARP1 inhibition radiosensitizes HNSCC cells deficient in homologous recombination by disabling the DNA replication fork elongation response. Oncotarget 2016;7:9732-41. 
28. Heeke AL, Pishvaian MJ, Lynce F, et al. Prevalence of Homologous Recombination-Related Gene Mutations Across Multiple Cancer Types. JCO Precis Oncol 2018;2018:PO.17.00286.

29. Djordjevic B, Hennessy BT, Li J, et al. Clinical assessment of PTEN loss in endometrial carcinoma: immunohistochemistry outperforms gene sequencing. Mod Pathol 2012;25:699-708.

30. Forster MD, Dedes KJ, Sandhu S, et al. Treatment with olaparib in a patient with PTEN-deficient endometrioid endometrial cancer. Nat Rev Clin Oncol 2011;8:302-6.

31. Dedes KJ, Wetterskog D, Mendes-Pereira AM, et al. PTEN deficiency in endometrioid endometrial adenocarcinomas predicts sensitivity to PARP inhibitors. Sci Transl Med 2010;2:53ra75.

32. Mendes-Pereira AM, Martin SA, Brough R, et al. Synthetic lethal targeting of PTEN mutant cells with PARP inhibitors. EMBO Mol Med 2009;1:315-22.

33. Bryant HE, Schultz N, Thomas HD, et al. Specific killing of BRCA2-deficient tumours with inhibitors of poly(ADPribose) polymerase. Nature 2005;434:913-7.

Cite this article as: Wang Q, Zhang F, Gao H, Xu Y. Successful treatment of a patient with brain metastases from endometrial cancer using Niraparib: a case report. Ann Palliat Med 2021;10(1):818-827. doi: 10.21037/apm-21-113
34. Robson M, Goessl C, Domchek S. Olaparib for Metastatic Germline BRCA-Mutated Breast Cancer. N Engl J Med 2017;377:1792-3.

35. Kaufman B, Shapira-Frommer R, Schmutzler RK, et al. Olaparib monotherapy in patients with advanced cancer and a germline BRCA1/2 mutation. J Clin Oncol 2015;33:244-50.

36. Swisher EM, Lin KK, Oza AM, et al. Rucaparib in relapsed, platinum-sensitive high-grade ovarian carcinoma (ARIEL2 Part 1): an international, multicentre, openlabel, phase 2 trial. Lancet Oncol 2017;18:75-87.

37. Litton JK, Rugo HS, Ettl J, et al. Talazoparib in Patients with Advanced Breast Cancer and a Germline BRCA Mutation. N Engl J Med 2018;379:753-63.

38. Gockley AA, Kolin DL, Awtrey CS, et al. Durable response in a woman with recurrent low-grade endometrioid endometrial cancer and a germline BRCA2 mutation treated with a PARP inhibitor. Gynecol Oncol 2018;150:219-26.

(English Language Editor: J. Reynolds) 